\title{
Dos posibles recintos campamentales romanos en la provincia de Lugo: crítica y elogio de la noticia arqueológica*
}

\section{Two possible Roman camps in the province of Lugo: criticism and praise of the archaeological news}

\author{
Brais X. Currás Refojos \\ Instituto de Historia. CSIC \\ brais.curras@cchs.csic.es - ORCID iD: https://orcid.org/0000-0002-8975-070X \\ Esperanza Martín-Hernández \\ Dolabra Arqueológica \\ dolabra@dolabra.es - ORCID iD: https://orcid.org/0000-0003-1718-7281 \\ Luis F. López-González \\ Terra Arqueos S. L. \\ terraarqueos@hotmail.com - ORCID iD: https://orcid.org/0000-0001-9721-4643 \\ Joaquín Granados Castro \\ kino_gc@hotmail.com - ORCID iD: https://orcid.org/0000-0003-0858-068X
}

Enviado: 15-02-2021. Aceptado: 04-05-2021. Publicado online: 28-07-2021

Cómo citar este artículo / Citation: Currás Refojos, B. X., Martín-Hernández, E., López-González, L. F. y Granados Castro, J. (2021). "Dos posibles recintos campamentales romanos en la provincia de Lugo: crítica y elogio de la noticia arqueológica”. Archivo Español de Arqueología, 94, e13.

DOI: https://doi.org/10.3989/aespa.094.021.13

RESUMEN: Se presentan las evidencias de dos posibles recintos campamentales romanos situados en la provincia de Lugo y se analiza su morfología con medios de teledetección y prospección. Desde un punto de vista crítico, se reflexiona sobre las limitaciones y posibilidades de la noticia arqueológica como parte del proceso de generación de conocimiento histórico.

\footnotetext{
La investigación recogida en este artículo se ha llevado a cabo en el marco de los proyectos "LOKI: Economías locales, economía imperial: el Occidente de la Península Ibérica (siglos II a. C.- II d. C.)" (PID2019-104297GB-I00); "AVRARIA: el oro de Hispania. Impacto territorial, económico y medioambiental de la minería del oro en el Imperio romano" (2019-T1/HUM-14288; Consejería de Ciencia, Universidades e Innovación, Comunidad de Madrid); "MILITRANSFER: Paisaje y territorio militarizado en la Hispania romana: movilidad y transferencia cultural (ss. II a. C.-IV d. C.)” (HAR2017-85929-P) (MINECO/AEI/FEDER) y "NyNO: Paisajes culturales en el norte y noroeste de la Península Ibérica: territorios urbanos, agrarios y mineros" (202010E147).
} 
Palabras clave: Ejército romano; Arqueología del paisaje; teledetección; fotografía aérea; LiDAR; Gallaecia; Hispania.

ABSTRACT: Here we present the evidences of two possible Roman camps located in the province of Lugo and their morphology is analysed with remote sensing and field survey. From a critical point of view, we reflect on the limitations and possibilities of archaeological news as part of the process of generating historical knowledge.

Keywords: Roman Army; Landscape Archaeology; remote sensing; aerial photography; LiDAR; Gallaecia; Hispania.

Copyright: $\odot 2021$ CSIC. Este es un artículo de acceso abierto distribuido bajo los términos de la licencia de uso y distribución Creative Commons Reconocimiento 4.0 Internacional (CC BY 4.0).

\section{INTRODUCCIÓN}

En los últimos diez años se ha incrementado de forma notable la identificación de nuevos recintos campamentales en el cuadrante noroccidental de la península ibérica (Didierjean, Morillo y Petit-Aupert, 2014; Orejas et al., 2015; Costa, Fonte y Gago, 2019; Martín et al., 2020; Morillo et al., 2021). La popularización de los medios de teledetección, el desarrollo de la tecnología LiDAR y la simplificación del acceso a la fotografía aérea y a la imagen satélite, han supuesto una democratización en el manejo de la arqueología aérea. Tanto investigadores como aficionados pueden acceder ahora con facilidad a medios con los que hace dos o tres décadas tan solo trabajaba un número muy limitado de grupos de investigación. De este modo, hoy asistimos a una suerte de carrera por la identificación de nuevos indicios y evidencias de estructuras campamentales, que viene a llenar el vacío dejado por el subdesarrollo en la aplicación de medios de teledetección en la investigación arqueológica española. La arqueología aérea, a pesar de estar plenamente consolidada desde hace más de 40 años en la investigación europea (Maxwell y Wilson, 1987), tuvo sin embargo un eco mucho más limitado en el estudio de la presencia del ejército en el noroeste hispano (Loewinsohn, 1965; Sánchez-Palencia, 1986). Lamentablemente, todo este nuevo desarrollo del mapa de la actividad militar romana no ha encontrado un correlato en forma de un incremento parejo en la comprensión histórica del proceso de conquista y posterior control de las comunidades sometidas por Roma.

En este artículo presentamos dos posibles nuevos campamentos situados dentro del conventus Lucensis (Fig. 1) a modo de pequeña aportación con la que sentar las bases para futuros trabajos que permitan ampliar el conocimiento y entender los tiempos y las formas de los procesos complejos de conquista, dominación y explotación, llevados a cabo por el imperio romano y sus ejércitos. Con la publicación de estos dos recintos pretendemos reivindicar el papel de la noticia arqueológica como una herramienta útil, capaz de aportar de forma ágil nuevos datos a la comunidad científica, pero a la vez, queremos reflexionar sobre el alcance del descubrimiento vacío de contenido histórico.

\section{NUEVAS EVIDENCIAS CAMPAMENTALES}

\subsection{Alto da Cepeira}

El posible recinto campamental de Alto da Cepeira (Guitiriz, Lugo) se sitúa sobre un cerro que despunta sobre la margen derecha del río Ladra, subsidiario del Miño, en una posición desde la que se ejerce un claro dominio sobre uno de los valles que da acceso a la comarca de A Terra Chá. Fue identificado en 2016 por Joaquín Granados, a la vez que otras estructuras castrenses como O Penedo dos Lobos. El análisis del sitio con medios de teledetección revela la existencia de un recinto con planta de tendencia rectangular con las esquinas redondeadas, pero marcadamente irregular, que ocupa una superficie total de 5,2 ha (Figs. 2 y 3 ) y cuyo perímetro es de $880 \mathrm{~m}$. En las zonas mejor conservadas, el cierre perimetral se presenta en forma de loma con una altura máxima conservada de $1 \mathrm{~m}$ y un ancho máximo aproximado de $6 \mathrm{~m}$, que parece corresponder a un agger (Fig. 4). En el esquinal suroeste y sobre todo en el lateral norte, el recinto se forma parcialmente aprovechando los afloramientos rocosos de esquisto. No se aprecian indicios suficientemente claros de la existencia de un foso, ni sobre el terreno, ni con teledetección. En prospección, bajo unas condiciones óptimas de visibilidad después de que toda la superficie del recinto fuese desbrozada, no se documentó ningún resto material. Los vecinos del lugar, que trabajaron estas tierras, tampoco mencionan la aparición de restos al arar. 


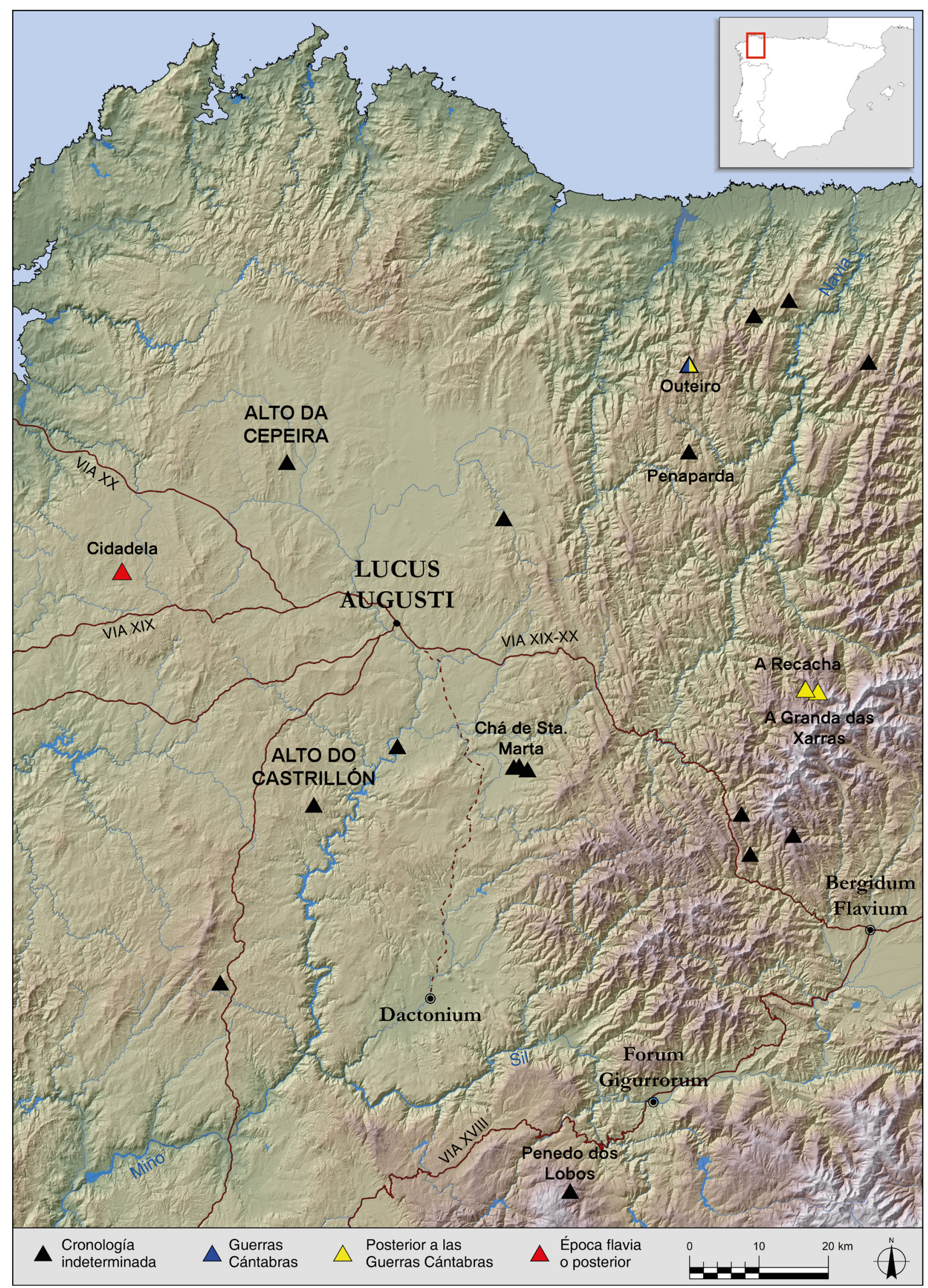

Figura 1. Evidencias campamentales en el área lucense (elaboración propia). 


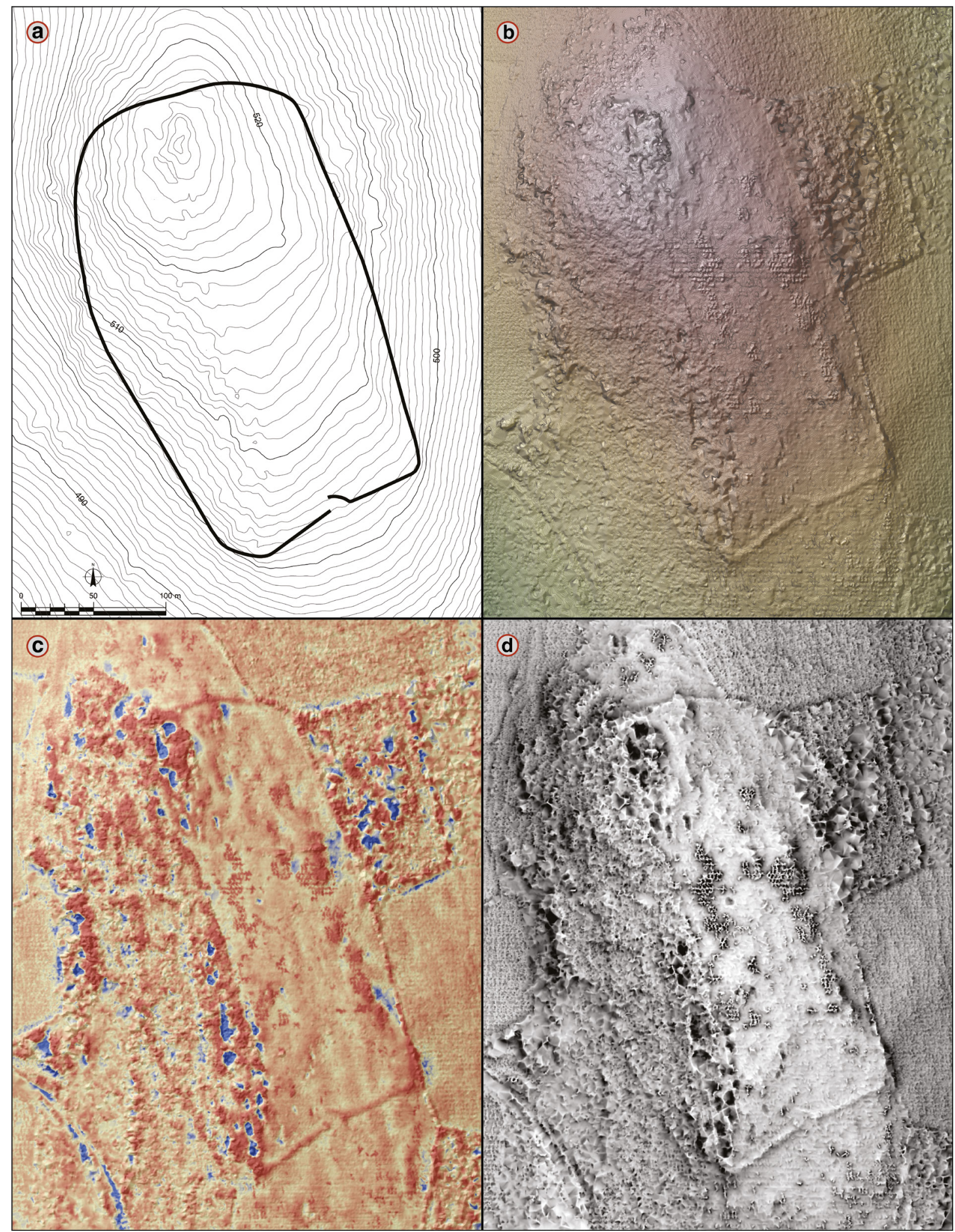

Figura 2. El recinto de Alto da Cepeira: a) fotointerpretación; b) topografía (curvas de nivel cada $10 \mathrm{~cm}$ ); c) representación del recinto aplicando local relief model; d) representación del recinto aplicando sky-view factor (elaboración propia). 


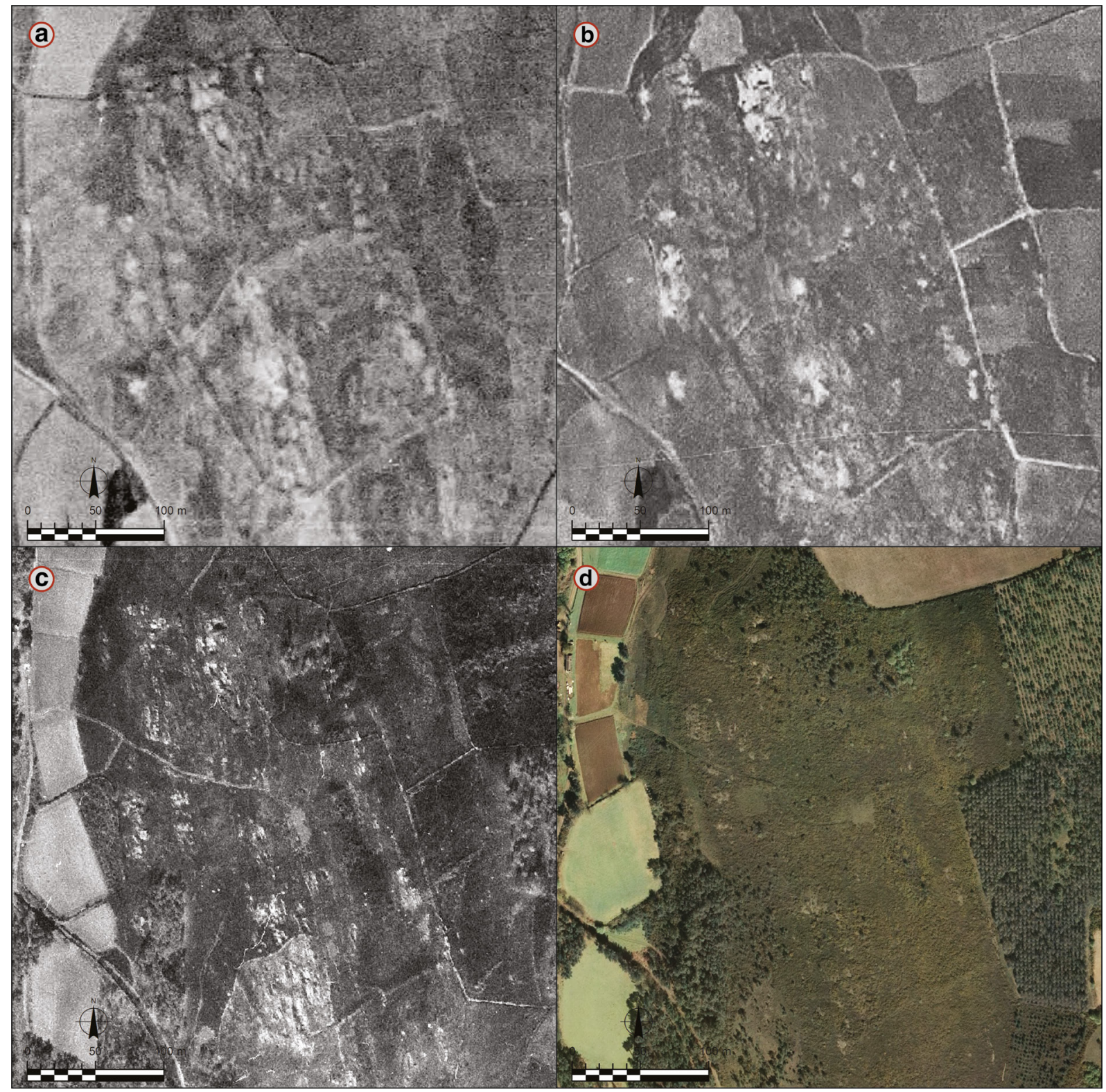

Figura 3. El recinto de Alto da Cepeira en la fotografía aérea histórica: a) vuelo americano de 1946; b) vuelo americano de 1956; c) vuelo Interministerial de 1973-1986; d) vuelo del PNOA de 2007 (elaboración propia).

Tanto en los vuelos de la USAF de los años 1946 y 1956 (series A y B), como en el vuelo interministerial (1973-1986) se puede apreciar la totalidad del perímetro del recinto (Fig. 3), si bien es cierto que la mitad occidental aparece especialmente alterada ya en los años 40 por trabajos de extracción de piedra ("lousa"), que fue empleada en la construcción de los límites del parcelario. El flanco oriental, por su parte, sirve de base a la cerca que funciona como linde, que aprovecha la elevación del agger para el cierre. La fotografía aérea histórica muestra además la existencia de parcelaciones internas dentro del recinto. En todos los casos se trata de lindes modernas, pero en el extremo noroccidental se observa una posible división interna del recinto, que se aprecia sobre todo en las fotografías de los vuelos de 1946 y 1956. La topografía obtenida por LiDAR (Fig. 2), la realización de un vuelo con dron para la obtención de un modelo fotogramétrico y la aplicación de técnicas de representación como el sky-view factor y el local relief model (Kokalj y Somrak, 2019) confirman la entidad de las estructuras documentadas mediante fotografía aérea.

El recinto se asemeja en su traza al tipo de planta en forma de naipe, si bien adopta laterales algo irregu- 


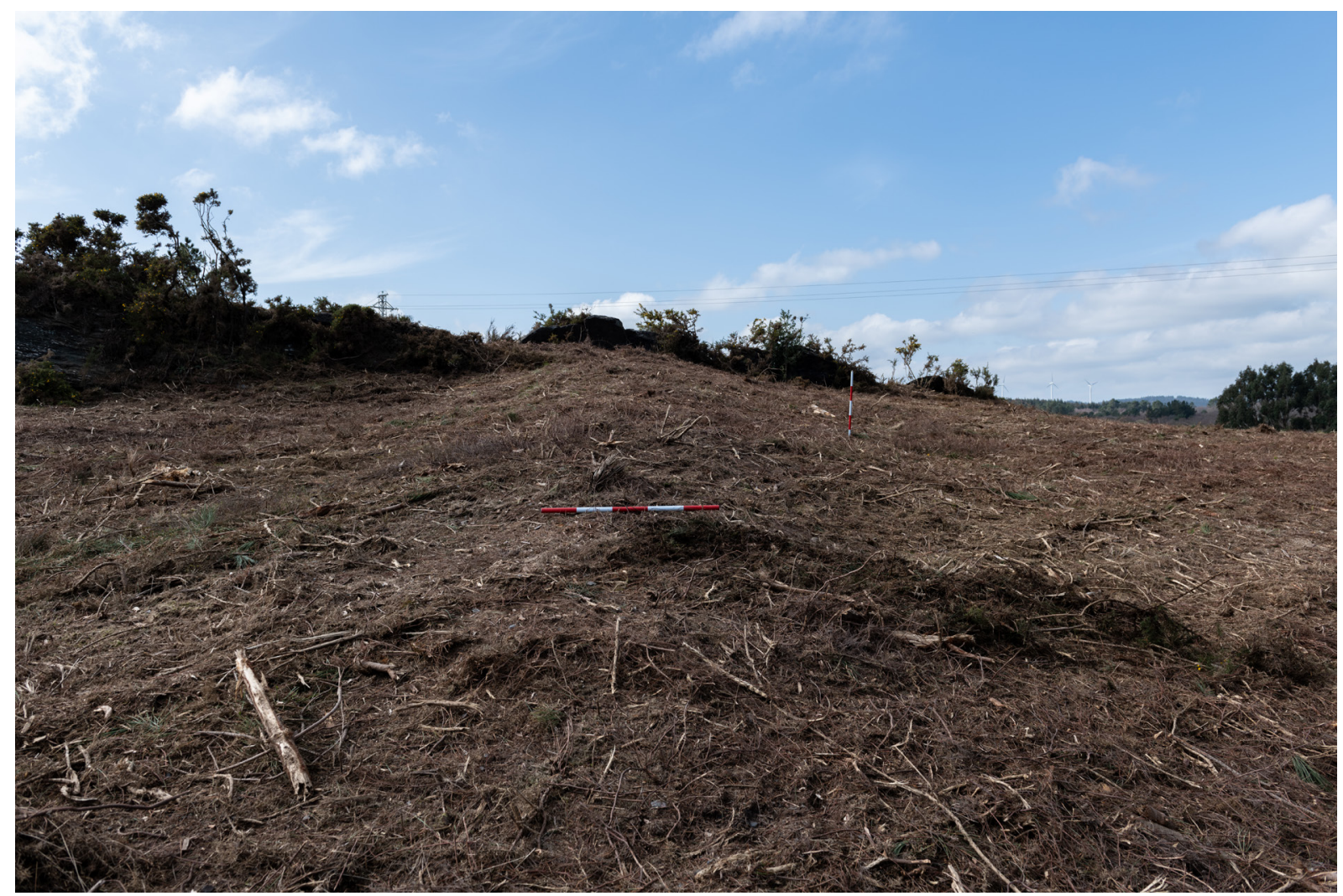

Figura 4. Vista del agger del extremo norte del Alto da Cepeira (fotografía de los autores).

lares por adaptación a la topografía de la loma en que se emplaza. En su eje mayor mide $305 \mathrm{~m}$, por $182 \mathrm{~m}$ en el eje menor, lo que implica un módulo constructivo de 5:3. Ninguno de los esquinales llega a formar exactamente un ángulo recto. Esta falta de adecuación al plano canónico de los recintos castrenses podría explicarse quizás por su construcción ajustada a la falda del cerro y por las dificultades que presenta la zona occidental del mismo, en donde se observa una notable concentración de afloramientos rocosos.

Uno de los elementos más característicos del recinto es el acceso en clavícula que se observa claramente en el cierre meridional, con un trazado en cuarto de círculo, y que se aprecia con nitidez tanto en la fotografía aérea histórica, como en los modelos del terreno obtenidos con datos LiDAR y por fotogrametría (Fig. 5). La entrada se realiza a través de una especie de rampa que aprovecha un canchal de esquisto, cuyo lateral aparece tallado, dando forma a algo que podríamos denominar como "contra clavícula" y que supondría una limitación añadida al acceso. La realización de una excavación en área permitirá concretar la caracterización formal de la entrada al recinto.

Los vecinos identifican este lugar como O Castro y justo al pie, por el oeste, se documenta el topónimo de
Roza Castro. Esta denominación plantea interesantes cuestiones sobre el modo en que empleamos la toponimia como indicador en la prospección. La toponimia es inespecífica desde un punto de vista arqueológico e histórico, aunque al mismo tiempo es extremadamente precisa en la relación analógica que establece para describir morfológicamente los elementos concretos que representan una alteración o una discontinuidad significativa del paisaje para las comunidades que lo habitan y lo nombran. En este caso, vemos que con "Castro" no se hace referencia a un asentamiento de la Edad del Hierro tipo castro, sino que, de un modo más genérico, se describe un recinto fortificado situado en una posición elevada.

\subsection{Alto do CASTRILlón}

El sitio de Alto do Castrillón se encuentra en un cerro que sobresale en el paisaje, situado justo en el límite entre los municipios de Taboada y Portomarín. Se trata de un recinto de forma rectangular y esquinas redondeadas, solo parcialmente conservado (Fig. 6), que fue identificado dentro del trabajo de análisis sistemático del territorio llevado a cabo en la tesis doctoral de L. F. López González (2020, p. 194 y Anexo 


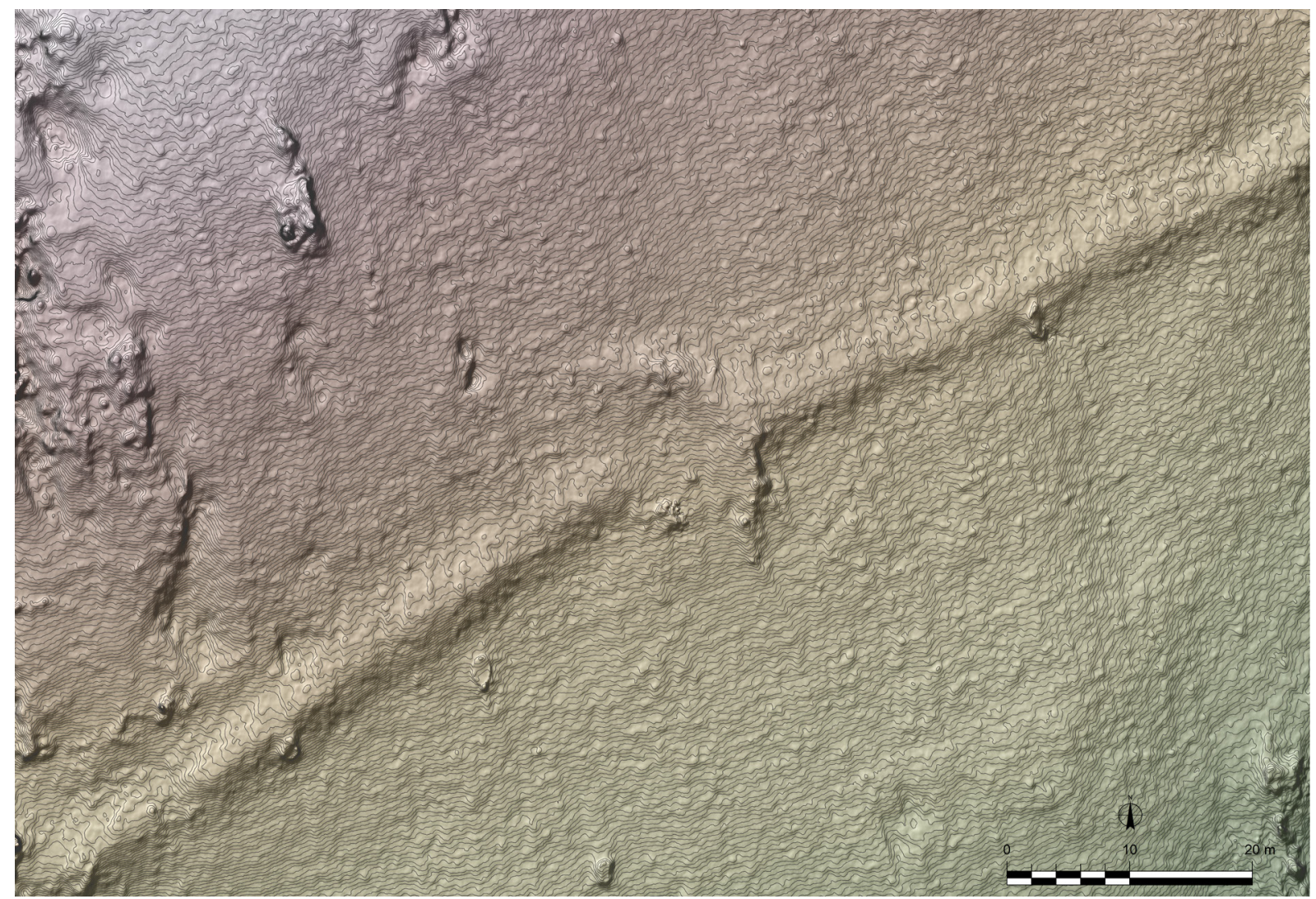

Figura 5. Topografía de la entrada en clavícula al recinto de Alto da Cepeira. Modelo del terreno obtenido por fotogrametría, con curvas de nivel cada $5 \mathrm{~cm}$ (elaboración propia).

1a, p. 507). Su morfología es reconocible únicamente en el vuelo americano de 1956, donde se aprecia lo que parece ser una estructura campamental con la típica forma de naipe, de la que se pueden identificar tan solo unos $450 \mathrm{~m}$ lineales, correspondientes a la mitad suroriental del recinto. En el punto central y más elevado del cerro se sitúa un túmulo, conocido como A Medorra. Sobre la imagen se detecta un crecimiento diferencial de vegetación, que aparentemente se corresponde con el foso. Su trazado es claramente discordante respecto al parcelario y las lindes observables en la fotografía en ningún punto coinciden con su estructura, hecho que acredita una antigüedad relativa respecto a las divisiones de las tierras. La entidad de los restos observables a través del análisis estereoscópico de la fotografía aérea del año 1956 es modesta, pero el topónimo "Castrillón" indudablemente hace referencia a una estructura más o menos destacada sobre el entorno, que en algún momento tuvo que ser fácilmente reconocible sobre el terreno y que hoy aparece totalmente desdibujada por el cultivo de la tierra y las repoblaciones forestales.

La definición de la posible estructura castrense del Alto do Castrillón mediante teledetección presenta notables dificultades. En el vuelo americano de 1946 no se distingue ninguna traza del recinto a pesar de que en ese momento todo el monte estaba en cultivo y las condiciones eran óptimas para poder reconocer las marcas dejadas por el crecimiento diferencial de la vegetación. La escasa definición de la película de este vuelo podría explicar los problemas para observar huellas claras. El examen mediante visión estereoscópica del vuelo americano de 1956 sí permite identificar con claridad los trazos del posible campamento, pero en las fotografías posteriores vuelven a desaparecer. Tanto en el vuelo interministerial (1973-1986) como en el nacional (1980-1986) se aprecia que la zona ya no estaba en cultivo y estaba ocupada por monte bajo, lo que obstaculiza el reconocimiento de las marcas sobre el suelo. En cambio, en el vuelo del SIGPAC (19972003) sí se consigue observar la zona de la esquina SO del recinto, no sin dificultad. Posteriormente, los trabajos de repoblación forestal, que ya estaban en marcha en ese momento y que se pueden apreciar en los siguientes vuelos del PNOA, terminaron por arrasar cualquier vestigio de las estructuras, que tampoco son identificables ya en prospección sobre el terreno. Por su parte, los datos LiDAR del PNOA no regis- 


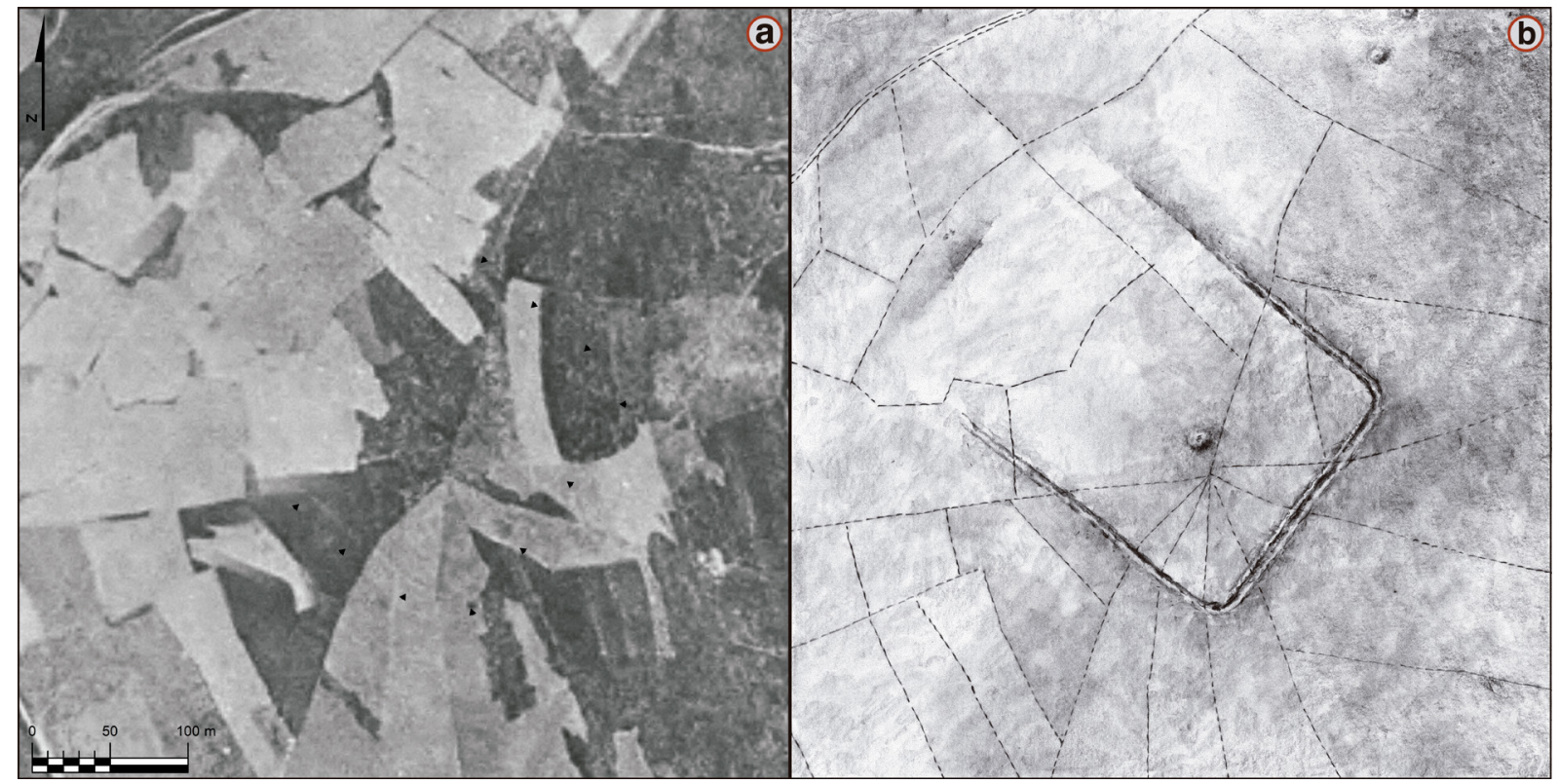

Figura 6. El recinto de Alto do Castrillón: a) vuelo americano de 1956; b) fotointerpretación (elaboración propia).

tran señal alguna de un recinto cuyos últimos restos habrían desaparecido años antes de la realización del vuelo (2009). Todo esto abunda en la necesidad de analizar secuencialmente diferentes vuelos históricos, y de contar con el LiDAR como una herramienta complementaria con otros medios de teledetección.

\section{DE LA CONQUISTA A LA DOMINACIÓN. CONSIDERACIONES CRONOLÓGICAS SOBRE LOS CAMPAMENTOS DEL ÁREA LUCENSE}

Es poco lo que puede apuntarse sobre la incardinación cronológica de los recintos documentados en Alto da Cepeira y Alto do Castrillón sin una intervención sobre el terreno más exhaustiva. Su estructura, su probable asimilación a la planta en forma de naipe habitual en los campamentos altoimperiales o la entrada en clavícula, remiten inequívocamente a una cronología romana. $\mathrm{La}$ aparente escasa entidad de las estructuras de delimitación y la falta de hallazgos cerámicos en una primera aproximación, sugieren además que estamos ante establecimientos de carácter temporal. No obstante, empleando solo medios de teledetección y prospección en superficie resulta imposible avanzar más en cuestiones cronológicas y discriminar un posible encuadre dentro de los episodios bélicos de la conquista, en momentos inmediatamente posteriores de la "paz armada" (Morillo, 2017) o en el largo período en el que las tropas de Roma permanecieron acuarteladas en el noroeste hispano.

Las referencias cronológicas procedentes de las escasas intervenciones arqueológicas llevadas a cabo en los campamentos de carácter temporal del área lucense dibujan un panorama diverso. Las dataciones resultantes de la excavación de A Recacha y A Granda das Xarras apuntan hacia una ocupación situada en un momento posterior a las guerras cántabras (Orejas et al., 2015). En Outeiro (Menéndez y Sánchez, 2018), las fechas obtenidas por datación radiocarbónica presentan un intervalo cronológico amplio, que tanto puede relacionarse con las guerras como con la fase ulterior de control del territorio, si bien es cierto que con una mayor probabilidad estadística a $1 \sigma$ de situarse en un momento posterior al cambio de era. En la zona astur, las intervenciones en La Carisa sí apuntan claramente a los episodios bélicos que tienen lugar en el período de las guerras cántabras (Camino, 2015). La excavación del campamento de Penaparda (Costa et al., 2020) dio como resultado una datación situada en torno al s. XV d. C. que quizás se explique más por el tipo de material datado que por un verdadero origen moderno. Las dataciones de $\mathrm{C} 14$ proceden de los "estratos sobre los que se asentaba el mismo parapeto", por lo que deberían haber aportado un referente post quem más o menos claro. Sorprende en todo caso la afirmación de los autores de que nos situamos en un "horizonte cronológico anterior a los siglos V-VI d. C." (Costa et al., 2020, p. 68). El campamento de Penedo dos Lobos se ha relacionado con las guerras cántabras por la aparición en prospección de dos monedas de Publio Carisio datadas en el 25-22 a. C. (Costa, Fonte y Gago, 2019, p. 38), en lo que es una 
propuesta quizás algo aventurada, pues es evidente que el período de circulación del numerario -también en campamentos de campaña- puede exceder en décadas al momento de acuñación. Valga como ejemplo el denario de L. Piso Frugi del 90 a. C. documentado en el campamento de L.lagüezos, perteneciente al período bélico de las guerras cántabras (Martín y Camino, 2013, p. 273).

Hoy parece claro que el proceso de conquista es más amplio que el período que las fuentes escritas atribuyen a las guerras cántabras, y que se solapa con una fase más amplia durante la que se ponen en marcha los mecanismos de control y reestructuración territorial que cimientan las bases de la nueva sociedad provincial (Sastre et al., 2017). Lo vemos por ejemplo en la permanencia de las tropas en los campamentos de La Loma, inicialmente adscritos a un episodio de asedio durante las guerras (Peralta, 2008), pero en donde la identificación de monedas posteriores al 12 a. C. (Gómez Barreiro, 2012, p. 216, nota 13; cfr. Peralta, Hierro y Gutiérrez, 2011) demuestra la existencia de guarniciones al menos una década después de finalizar el conflicto, algo ya sugerido años antes (Morillo, 2008, p. 148-149). La identificación de monedas de la ceca de Caesaraugusta en el campamento de Campo de las Cercas (Peralta, 2002, p. 237), también datadas del 12 a. C. en adelante, incide sobre esta misma idea. El relato que nos llega de la conquista es eminentemente propagandístico y reproduce una imagen a medida de la proclamación de Augusto como responsable de la nueva pax. Sabemos, no obstante, que el comienzo de la conquista del noroeste es anterior a las guerras cántabras $(\mathrm{Cu}-$ rrás, 2019) y que se extiende en el tiempo a través de diversas estrategias de sometimiento de los vencidos. Las visiones reduccionistas que han asimilado toda evidencia campamental de carácter temporal a las guerras cántabras han sido objeto de duras críticas (Orejas et al., 2019; Morillo et al., 2020). A menudo, se ha caído en la simplificación de explicar la presencia del ejército romano en relación con un "teatro de operaciones" en el que es necesario reconstruir los movimientos de tropas y las líneas de avance, lo que no deja de ser nada más que un remedo de las viejas aproximaciones filológicas, revestidas ahora de una pátina de renovación tecnológica. Las dataciones resultantes de diferentes proyectos de investigación arrojan, por el contrario, un panorama mucho más complejo (Orejas et al., 2015). Terminadas las guerras, las tropas de Roma continuaron desplazándose por el territorio, desplegando los medios de control necesarios para que la conquista se materializase en forma de una dominación y una explotación duradera de los pueblos sometidos. La presencia militar no se limitó además a los ámbitos campamentales, sino que en varios casos fue penetrando también en asentamientos indígenas (Villa, 2009).

Roma empleó a sus ejércitos en el reconocimiento y exploración del territorio. Su papel fue determinante en las tareas de agrimensura, en el inventario de los recursos y en la elaboración de los censos (Nicolet, 1988), así como en la fundamental tarea de configuración territorial en civitates. Su actividad fue especialmente intensa en las zonas mineras (Sánchez-Palencia y Currás, 2015; Currás y Sánchez-Palencia, e. p.) y las tropas participaron también en la definición y trazado del sistema viario, ya en funcionamiento, al menos parcialmente, desde época de Augusto (Rodríguez Colmenero, Ferrer y Álvarez, 2004). Por todo ello, a la hora de explicar sitios como Alto da Cepeira o Alto do Castrillón, no podemos caer en la ingenuidad de atribuir necesariamente toda la actividad militar romana a episodios bélicos y movimientos de tropas dentro de las llamadas guerras cántabras. Los cometidos de las tropas de Roma fueron múltiples y sabemos que fueron continuados en el tiempo.

\section{CONCLUSIONES}

La noticia arqueológica es un texto breve, eminentemente descriptivo, que aporta datos sustanciales sobre novedades relevantes en la investigación. Como tal, la noticia puede ser un instrumento útil, versátil, que permite sacar a la luz rápidamente avances y resultados de trabajos de campo, aportaciones cronológicas de alcance, hallazgos materiales singulares... La arqueología militar se ha beneficiado de este formato y en los últimos años se han dado a conocer nuevos recintos campamentales mediante trabajos centrados en la descripción formal del sitio, desde una concepción de la noticia como base para futuros estudios más detallados (p. ej. Cordero, Cerrillo y Pereira, 2017).

A lo largo de la última década, han proliferado los hallazgos y la publicación de recintos castrenses en el cuadrante noroccidental de la península ibérica. El mapa de la presencia militar en Asturia et Gallaecia se ha ido completando progresivamente con nuevos descubrimientos que, sin embargo, solo en contadas ocasiones han dado lugar al desarrollo de proyectos de investigación o reflexiones de conjunto (Palao, 2014; Morillo, 2016). La necesaria noticia no suple la imprescindible reflexión histórica, la interpretación y el desarrollo de proyectos de intervención arqueológica sobre el terreno. La mayor parte de los hallazgos y de los trabajos derivados de ellos, salvo excepciones (Orejas et al., 2015; Menéndez y Sánchez, 2018; Camino, 2015; Currás y Sánchez-Palencia, e. p.), se mueven además en una absoluta imprecisión cronológica 
y a menudo incluso plantean problemas respecto a su identificación, clasificación y caracterización formal.

No debemos renunciar a la noticia arqueológica. La incorporación de nuevos datos nunca es desdeñable, pero la identificación de más sitios militares debiera dejar de ser cuanto antes un objetivo prioritario de la investigación, para dar paso a su documentación $\mathrm{y}$ excavación, y al desarrollo de proyectos que hagan posible su comprensión dentro de los procesos de transformación derivados de la sumisión del noroeste bajo el dominio de Roma. Urge superar la concepción del recinto campamental como un hecho aislado, para entenderlo en su contexto territorial, en relación al poblamiento local, a las estructuras viarias y a las formas de explotar y controlar la tierra. Todas las noticias que están dando a conocer nuevas evidencias campamentales son innegablemente útiles, pero debemos reconocer que no son más que un primer paso hacia una arqueología de la conquista, en gran medida todavía pendiente (Orejas y Sánchez-Palencia, 1999). Con esta breve publicación de los dos posibles campamentos de Alto da Cepeira y Alto do Castrillón queremos reivindicar la validez de la noticia cruda, descriptiva y objetiva, que sirve como inicio de una investigación y no como su objetivo.

\section{BIBLIOGRAFÍA}

Camino Mayor, J. (2015). "La línea de operaciones de la vía Carisa (Asturias y norte de León)”. En: Camino Mayor, J., Peralta labrador E. y Torres Martínez J. F. (Coords.). Las Guerras Astur-Cántabras. Gijón: Ayuntamiento de Gijón, pp. 217-237.

Cordero Ruiz, T., Cerrillo Cuenca, E. y Pereira, C. (2017). "Detección de un nuevo campamento romano en las inmediaciones de Mérida mediante tecnología LiDAR". Saguntvm, 49, pp. 197-201. DOI: https://doi.org/10.7203/sagvntvm.49.10025

Costa, J., Fonte, J. y Gago, M. (2019). "The reassessment of the roman military presence in Galicia and northern Portugal through digital tools: archaeological diversity and historical problems". Mediterranean Archaeology and Archaeometry, 19 (3), pp. 17-49.

Costa, J., Menéndez, A., Fonte, J. y Alonso, F. (2020). “A Penaparda (A Fonsagrada, Lugo - Santalla, Asturias): intervención arqueológica en un recinto campamental romano en el occidente de la cordillera cantábrica". Cuadernos de Estudios Gallegos, 67 (133), pp. 45-74. DOI: https://doi.org/10.3989/ceg.2020.133.02

Currás Refojos, B. X. (2019). Las sociedades de los castros entre la Edad del Hierro y la dominación de Roma: estudio del paisaje del Baixo Miño. Bibliotheca Praehistorica Hispana, 35. Madrid: CSIC.

Currás Refojos, B. X. y Sánchez-Palencia, F. J. (en prensa). "Landscape archaeology of Roman gold mining in Lusitania: The 'Aurifer Tagus' project". Antiquity.

Didierjean, F., Morillo, A. y Petit-Aupert, C. (2014). “Traces de guerres, traces de paix armée: l'apport de quatre campagnes de prospection aérienne dans le nord de l'Espagne”. En: Cadiou, F. y Navarro, M. (Eds.). La guerre et ses traces. Conflicts et Sociétés en His-panie à l'époque de la conquête romaine (IIIe-Ier siècle av. J. C.). Bordeaux: Ausonius, pp. 149-179.

Gómez Barreiro, M. (2012). "Circulación monetaria en la antigüedad en la provincia de Palencia”. En: Fernández Ibáñez, C. y Bohigas Roldán, R. (Coords.). In durii regione romanitas. Estudios sobre la presencia romana en el valle del Duero en homenaje a Javier Cortés Álvarez de Miranda. Palencia-Santander: Diputación Provincial de Palencia, pp. 211-222.

Kokalj, Ž. y Somrak, M. (2019). "Why Not a Single Image? Combining Visualizations to Facilitate Fieldwork and On-Screen Mapping”. Remote Sensing, 11(7), pp. 747.

Loewinsohn, E. (1965). "Una calzada y dos campamentos romanos del Conventus Asturum". Archivo Español de Arqueología, 38, pp. 26-43.

López-González, L. F. (2020). Castros y territorio: evolución del poblamiento castreño y de los procesos de territorialización en la sierra oriental gallega y sector meridional lucense. Tesis doctoral inédita. Universidad Complutense de Madrid.

Maxwell, G. S. y Wilson, D. R. (1987). "Air Reconnaissance in Roman Britain 1977-84”. Britannia, 18, pp. 1-48. DOI: https://doi.org/10.2307/526438

Martín-Hernández, E. y Camino Mayor, J. (2013). “El Picu L.l.agüezos, un nuevo campamento romano en la vía Carisa". En: Excavaciones arqueológicas en Asturias 20072012. Oviedo: Principado de Asturias, pp. 267-276.

Martín-Hernández, E., Martínez-Velasco, A., Díaz-Alonso, D., Muñoz-Villarejo, F. y Bécares, L. (2020). "Castrametación romana en la Meseta Norte hispana: nuevas evidencias de recintos militares en la vertiente meridional de la cordillera Cantábrica (provincias de Burgos y Palencia)". Zephyrus, 86, pp. 143-164.

DOI: https://doi.org/10.14201/zephyrus202086143164

Menéndez Granda, A. y Sánchez Hidalgo, E. (2018). “Campaña de sondeos arqueológicos en el campamento de época romana del Pico El Outeiro Zarrado (Taramundi-Villanueva de Oscos)". En: Excavaciones arqueológicas en Asturias 2013-2016. Oviedo: Principado de Asturias, pp. 283-292.

Morillo, Á. (2008). "La implantación militar romana en el territorio de Cantabria durante el Alto Imperio". En: Aja, J. R., Cisneros, M. y Ramírez, J. L. (Eds.). Los cántabros en la Antigüedad. La historia frente al mito. Santander: Universidad de Cantabria, pp. 142-154.

Morillo, Á. (2016). "El territorio galaico durante las guerras cántabras: nuevas perspectivas". En: Morais, R., Bandeira, M. y Sousa, M. J. (Eds.). Celebração do Bimilenário de Augusto. Ad nationes. Ethnous Kallaikon. Braga: Câmara Municipal de Braga, pp. 124-135.

Morillo, Á. (2017). "El periodo de la «Paz Armada» en el norte de Hispania (19/15 a.C.-15/20 d.C.): ¿la creación de un sistema de defensa sin frontera?". Gerión, 35, pp. 191-223. DOI: https://doi.org/10.5209/geri.56144

Morillo, Á., Adroher, A., Dobson, M. y Martín Hernández, E. 
(2020). "Constructing the archaeology of the Roman conquest of Hispania: new evidence, perspectives and challenges". Journal of Roman Archaeology, 33, pp. 36-52. DOI: https://doi.org/10.1017/s1047759420000902

Morillo, Á., Currás, B. X., Orejas, A. y Nobilini, A (2021). "El conjunto de campamentos romanos para prácticas de Trobajo del Camino (San Andrés de Rabanedo) y Oteruelo de la Valdoncina (León). Una aproximación preliminar". Gladius, 41, pp. 91-120.

Nicolet, C. (1988). L'inventaire du monde. Géographie et politique aux origines del'Empire romain. París: Fayard.

Orejas, A. y Sánchez-Palencia, F. J. (1999). “Arqueología de la Conquista del Noroeste de la Península Ibérica". En: Balbín, R. de y Bueno, P. (Eds.). II Congreso de Arqueología Peninsular (Zamora, del 24 al 27 de septiembre de 1996). Tomo IV: Arqueología Romana y Medieval. Alcalá de Henares-Zamora: Fundación Rei Afonso Henriques, pp. 23-37.

Orejas, A., Sánchez-Palencia, F. J., Beltrán, A., Ron, J. A., López, L. F.; Currás, B. X., Romero, D., Zubiaurre, E., Pecharromán, J. L. y Arboledas, L. (2015). "Conquista, articulación del territorio y explotación de recursos en el límite entre el convento lucense y el de los astures (Proyecto IVGA)". En: Camino Mayor, J., Peralta labrador E. y Torres Martínez J. F. (Coords.). Las Guerras Astur-Cántabras. Gijón: Ayuntamiento de Gijón, pp. 247-259.

Orejas, A., Sánchez-Palencia, F. J., Currás, B. X., Ron, J. A. y López, L. F. (2019). "Campamentos militares durante la primera ocupación romana del noroeste de la península Ibérica". En: Vallori Márquez, B., Rueda Galán, C. y Bellón Ruiz J. P. (Eds.). Accampamenti, guarnigioni e assedi durante la Seconda Guerra Punica e la conquista romana (secoli III-I a. C.): prospettive archeologiche. Roma: Quasar, pp. 97-111.

Palao Vicente, J. J. (2014). “Augusto y el ejército romano en la provincia de Hispania citerior. ¿Nuevas respuestas a viejos interrogantes?". Veleia, 31, pp. 53-78.

Peralta Labrador, E. J. (2002). "Castros y campamentos de campaña de las Guerras Cántabras". En: De Blas Cortina, M. A. y Villa Valdés, A. (Eds.). Los poblados fortifi- cados del Noroeste de la Península Ibérica: Formación y desarrollo de la cultura castreña. Coloquios de Arqueología en la Cuenca del Navia. Navia: Ayuntamiento de Navia, pp. 225-240.

Peralta Labrador, E. J. (2008). "La conquista romana de la Montaña Palentina: el asedio de La Loma (Santibáñez de la Peña)". Colección de Historia Montaña Palentina, 2, pp. 13-36.

Peralta Labrador, E. J., Hierro Gárate, J. A. y Gutiérrez Cuenca, E. (2011). "Las monedas de los campamentos romanos de campaña de las Guerras Cántabras del asedio de La Loma, Castillejo y El Alambre”. Lucentum, 30, pp. 151-172. DOI: https://doi.org/10.14198/lvcentvm2011.30.07

Rodríguez Colmenero, A., Ferrer Sierra, S y Álvarez Asorey, R. (2004). Callaeciae et Astvriae itinera romana. Miliarios e outras inscricións viarias romanas do noroeste hispánico (conventos bracarense, lucense e asturicense). Santiago de Compostela: Consello da Cultura Galega.

Sánchez-Palencia, F. J. (1986). "El campamento romano de Valdemeda, Manzaneda (León). Ocupación militar y explotación aurífera en el NW peninsular". Numantia, 2, pp. 227-235.

Sánchez-Palencia, F. J. y Currás, B. X. (2015). “Campamentos romanos en zonas mineras del cuadrante noroeste de la Península Ibérica”, En: Camino Mayor, J., Peralta labrador E. y Torres Martínez J. F. (Coords.). Las Guerras Astur-Cántabras. Gijón: Ayuntamiento de Gijón, pp. 273-283.

Sastre, I., Orejas, A., Currás, B. X. y Zubiaurre, E. (2017). "La formación de la sociedad provincial en el Noroeste hispano y su evolución: "civitates" y mundo rural". Gerión, 35 (2), pp. 537-552.

DOI: https://doi.org/10.5209/geri.59923

Villa Valdés, Á. (2009). “¿De aldea fortificada a Caput Civitatis? Tradición y ruptura en una comunidad castreña del siglo I d.C.: el poblado de Chao Samartín (Grandas de Salime, Asturias)". Cuadernos de Prehistoria y Arqueología Universidad Autónoma de Madrid, 35, pp. 7-26. DOI: https://doi.org/10.15366/cupauam2009.35.001 